\title{
Impact of the Coronavirus Disease Pandemic on Patients with Head Injuries in South Korea
}

\author{
Taek Min Nam, ${ }^{1}$ Do-Hyung Kim, ${ }^{2}$ Ji Hwan Jang, ${ }^{1}$ Young Zoon Kim, Kyu Hong Kim, ${ }^{1}$ Seung Hwan Kim \\ Department of Neurosurgery, Samsung Changwon Hospital, Sungkyunkwan University School of Medicine, Changwon, Korea \\ Department of Neurology, ${ }^{2}$ Samsung Changwon Hospital, Sungkyunkwan University School of Medicine, Changwon, Korea
}

Objective : The coronavirus disease 2019 (COVID-19) pandemic is affecting the characteristics of patients with head injuries. This study aimed to evaluate the effect of the COVID-19 pandemic on patients with head injuries at a regional emergency medical center in South Korea.

Methods : From April 2019 to November 2020, 350 patients with head injuries were admitted to our hospital. The study period was divided into the pre-COVID-19 ( $n=169)$ and COVID-19 ( $n=181)$ eras (10 months each). Patients with severe head injuries requiring surgery ( $n=74)$ were categorized into those who underwent surgery $(n=41)$ and those who refused surgery $(n=33)$.

Results : Head injuries in pediatric patients ( $<3$ years) were more frequent in the COVID-19 era than in the pre-COVID-19 era $(8.8 \%$ vs. $3.6 \%, p=0.048)$. More patients refused surgery in the COVID-19 era than in the pre-COVID-19 era $(57.9 \%$ vs. $30.6 \%, p=0.021)$. Refusal of surgery was associated with old age (67.7 \pm 14.5 vs. $52.4 \pm 19.1, p<0.001)$, marital status (married, $84.8 \%$ vs. $61.0 \%, p=0.037$ ), unemployment $(42.4 \%$ vs. $68.3 \%, p=0.034)$, COVID-19 era ( $66.7 \%$ vs. $39.0 \%, p=0.021)$, and lower Glasgow coma scale scores $(6.12 \pm 3.08$ vs. 10.6 $\pm 3.80, p<0.001)$. Multivariable logistic regression analysis revealed that refusal of surgery was independently associated with old age (adjusted odds ratio [OR], 1.084; 95\% confidence interval [CI], 1.030-1.140; $p=0.002$ ), COVID-19 era (adjusted OR, 6.869; 95\% $\mathrm{Cl}, 1.624-29.054 ; p=0.009$ ), and lower Glasgow coma scale scores (adjusted OR, 0.694; 95\% Cl, 0.568-0.848; $p<0.001$ ).

Conclusion : We observed an increased prevalence of head injuries in pediatric patients ( $<3$ years) during the COVID-19 pandemic. Additionally, among patients with severe head injuries requiring surgery, more patients refused to undergo surgery during the COVID-19 pandemic.

Key Words : Head injuries · SARS-CoV-2 · Coronavirus disease 2019 virus · COVID-19 virus · Korea.

\section{INTRODUCTION}

Severe acute respiratory syndrome coronavirus 2 (SARS$\mathrm{CoV}-2)$ is a novel corona-virus that causes coronavirus disease 2019 (COVID-19). The first case of COVID-19 was reported in Wuhan, Hubei province, China on December 2019 $9^{9,10,14)}$, following which the infection spread across China to other countries within few weeks. Finally, on January 30, 2020, the World Health Organization (WHO) declared the COVID-19 outbreak as a public health emergency of international concern. On February 11, 2020, the WHO named the infection COVID-19.

- Received : April 1, $2021 \cdot$ Revised : June 11, 2021 •Accepted : August 25, 2021

- Address for reprints : Seung Hwan Kim

Department of Neurosurgery, Samsung Changwon Hospital, Sungkyunkwan University School of Medicine, 158 Palyong-ro, Masanhoiwon-gu, Changwon 51353, Korea Tel : +82-55-233-5896, Fax : +82-55-233-6527, E-mail : aajechtkskim@hanmail.net, ORCID : https://orcid.org/0000-0002-5898-6517

This is an Open Access article distributed under the terms of the Creative Commons Attribution Non-Commercial License (http://creativecommons.org/licenses/by-nc/4.0) which permits unrestricted non-commercial use, distribution, and reproduction in any medium, provided the original work is properly cited. 
To restrict the spread of SARS-CoV-2, voluntary nationwide lockdowns were proposed and implemented worldwide, including restrictions on the movement of people and transportation, and prohibiting economic, educational, sporting, cultural, and religious activities ${ }^{1,2,4,6)}$. This lockdown was intended to delay the spread of the disease.

The COVID-19 pandemic is influencing almost every area of life ${ }^{8)}$. The COVID-19 pandemic and subsequent social distancing and lockdowns have had an impact on trauma patterns in patients. The spectrum of traumatic injuries has to shift from high-energy trauma such as road traffic accidents to low-energy trauma such as house-hold injuries in the elderly and pediatric populations, which may be partially attributed to the social distancing measures and lockdown ${ }^{5)}$. Similarly, the COVID-19 pandemic is changing the characteristics of patients with head injuries ${ }^{3)}$.

This study aimed to evaluate the effect of the COVID-19 pandemic on patients with head injuries and their management. We compared patients with head injuries between the pre-COVID-19 era and the COVID-19 eras. We also compared the treatment planning decisions for patients.

\section{MATERIALS AND METHODS}

\section{Ethics statement}

The present study protocol was reviewed and approved by the Institutional Review Board of Samsung Changwon Hospital (SCMC 2020-12-005). This type of study does not require informed consent.

\section{Study population}

We reviewed the data on patients with head injuries who were admitted to our hospital, a regional emergency medical center in South Korea, from April 2019 to November 2020. A total of 350 patients with head injuries who met the inclusion criteria were enrolled in this study. The inclusion criteria were as follows : 1) patients with acute head injuries who visited the emergency department and 2) patients admitted to the neurosurgery department.

\section{Data collection and patient characteristics}

The medical records and imaging data of the patients were reviewed. Data on age, sex, marital status, employment status, religion, injury type, hospital day, Glasgow coma scale (GCS) scores, Glasgow outcome scale (GOS) scores, operation agreement, do not resuscitate (DNR) agreement, and organ donation agreement.

The study period was divided into the pre-COVID-19 $(n=169)$ and COVID-19 ( $n=181)$ eras (10 months each). The pre-COVID-19 era was from April 2019 to January 2020 and the COVID-19 era was from February 2020 to November 2020. Marital status was categorized as "unmarried" and "married". Employment and religion were tabulated as "present" or "absent". Injury types were "motor vehicle collision (MVC)" and "non-MVC". The decision to undergo surgery and DNR agreement in patients with severe head injuries was family dependent due to impaired brain function. The decision-makers considered the patient's past wishes and beliefs, as well as the circumstances of the remaining family. In case of cardiac arrest in patients with DNR, cardiopulmonary resuscitation was not performed. The decision for organ donation was made by the family after declaration of brain death.

\section{Statistical analysis}

Variables were compared between the pre-COVID-19 and COVID-19 eras. Variables are also compared between those who decided to undergo surgery and those who refuse to undergo surgery among patients with severe head injuries requiring surgery. The chi-square test or Fisher's exact test was used to analyze the categorical variables. Student's t-test or the Mann-Whitney $U$ test was used for continuous variables. Multivariable analysis, including logistic regression, was used to evaluate factors affecting refusal of surgery. Variables with $p<0.05$ in univariate analysis were selected for the logistic regression model. A $p$-value of $<0.05$ was considered statistically significant. All analyses were performed using SPSS version 22 (IBM Corp., Armonk, NY, USA).

\section{RESULTS}

Of the 350 patients, 249 patients $(71.1 \%)$ were male. The mean patient age was 53.4 years. There were 107 unmarried and 243 married patients. There were 179 employed patients and 171 unemployed patients. Religions of the 138 patients (39.4\%) whose data was available were Catholicism $(n=12)$, Protestantism $(n=26)$, Buddhism $(n=97)$, and others $(n=3)$. 
COVID-19 and Head Injury Patients | Nam TM, et al.

Table 1. A comparison of the patients with head injuries between the pre-COVID-19 and COVID-19 eras

\begin{tabular}{|c|c|c|c|}
\hline & Pre-COVID-19 $(n=169)$ & COVID-19 $(n=181)$ & $p$-value \\
\hline Age (years) & $54.1 \pm 25.3$ & $52.8 \pm 26.7$ & 0.627 \\
\hline Age $<3$ years & $6(3.6)$ & $16(8.8)$ & 0.048 \\
\hline Age $<20$ years & $24(14.2)$ & $34(18.8)$ & 0.314 \\
\hline Sex, male & $118(69.8)$ & $131(72.4)$ & 0.638 \\
\hline Marital status, married & $119(70.4)$ & $124(68.5)$ & 0.729 \\
\hline Employment status, unemployed & $78(46.1)$ & $93(51.4)$ & 0.338 \\
\hline Religion & $59(34.9)$ & 79 (43.6) & 0.102 \\
\hline Catholicism & 5 & 7 & \\
\hline Protestantism & 13 & 13 & \\
\hline Buddhism & 39 & 58 & \\
\hline Others & 2 & 1 & \\
\hline Injury type, MVC & $44(26.0)$ & $52(28.7)$ & 0.632 \\
\hline Hospital day (days) & $15.8 \pm 15.9$ & $16.1 \pm 19.1$ & 0.867 \\
\hline GCS score & $13.1 \pm 3.0$ & $12.9 \pm 3.53$ & 0.550 \\
\hline GOS score & $4.27 \pm 1.37$ & $4.17 \pm 1.47$ & 0.509 \\
\hline Requiring surgery & $36(21.3)$ & $38(21.0)$ & 1.000 \\
\hline Death & $20(11.8)$ & $28(15.5)$ & 0.354 \\
\hline DNR & $17(10.1)$ & $26(14.4)$ & 0.255 \\
\hline Organ donation & $1(0.6)$ & $4(2.2)$ & 0.373 \\
\hline
\end{tabular}

Values are presented as mean \pm standard deviation or number (\%). COVID-19 : coronavirus disease 2019, MVC : motor vehicle collision, GCS : Glasgow coma scale, GOS : Glasgow outcome scale, DNR : do not resuscitate

Table 2. A comparison of the patients who underwent surgery with those who refused surgery among patients with severe head injuries requiring surgery

\begin{tabular}{|c|c|c|c|}
\hline & Underwent surgery $(n=41)$ & Refused surgery $(n=33)$ & $p$-value \\
\hline Age (years)* & $52.4 \pm 19.1$ & $67.7 \pm 14.5$ & $<0.001$ \\
\hline Sex, male & $35(85.4)$ & $25(75.8)$ & 0.375 \\
\hline Marital status, married* & $25(61.0)$ & $28(84.8)$ & 0.037 \\
\hline Employment status, unemployed* & $13(31.7)$ & $17(57.6)$ & 0.034 \\
\hline Religion & $16(39.0)$ & $16(48.5)$ & 0.483 \\
\hline Catholicism & 2 & 0 & \\
\hline Protestantism & 2 & 4 & \\
\hline Buddhism & 11 & 12 & \\
\hline Others & 1 & 0 & \\
\hline Injury type, MVC & $15(36.6)$ & $10(30.3)$ & 0.627 \\
\hline COVID-19 pandemic* & & & 0.021 \\
\hline Pre-COVID era & 25 & 11 & \\
\hline COVID era & 16 & 22 & \\
\hline GCS score & $7(6-13)$ & $7(4-13)$ & 0.220 \\
\hline Organ donation & $1(2.4)$ & $4(12.1)$ & 0.165 \\
\hline
\end{tabular}

Values are presented as mean \pm standard deviation, median (interquartile range), or number (\%). ${ }^{*}$ Variables for logistic regression model. MVC : motor vehicle collision, COVID-19: coronavirus disease 2019, GCS : Glasgow coma scale 
Table 3. Multivariable logistic regression analysis of the factors associated with refusing surgery among patients with severe head injuries requiring surgery

\begin{tabular}{lcccccc} 
& Coefficient beta & Standard error & Wald & Adjusted OR & Adjusted 95\% Cl & $p$-value \\
\hline Old age & 0.073 & 0.020 & 12.666 & 1.076 & $1.033-1.120$ & $<0.001$ \\
COVID-19 era & 1.806 & 0.613 & 8.687 & 6.087 & $1.831-20.229$ & 0.003 \\
\hline
\end{tabular}

OR : odds ratio, Cl : confidence interval, COVID-19: coronavirus disease 2019

The head injury types were as follows : concussion $(n=21$, $6.00 \%)$, skull fracture ( $\mathrm{n}=40,11.4 \%)$, epidural hematoma $(n=48,13.7 \%)$, subdural hematoma ( $n=101,28.9 \%)$, subarachnoid hemorrhage $(n=111,31.7 \%)$, intracerebral hematoma $(n=28,8.00 \%)$, and intraventricular hemorrhage $(n=1,0.28 \%)$. MVCs and non-MVCs were observed in 96 (27.4\%) and 254 (72.6\%) patients, respectively. The mean hospital days were 16.0 \pm 17.6 days (mean days \pm standard deviation). The overall

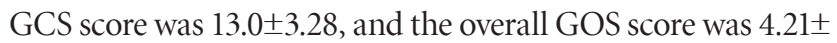
1.42. Among the 360 patients with head injuries, 74 patients (20.6\%) required surgery. Among these 74 patients, 41 patients (55.4\%) underwent surgery and 33 patients (44.6\%) refused surgery. The mortality rate was $13.7 \%(n=48) ; 43$ patients (89.6\%) died with DNR, and organ donation was performed in five patients (10.4\%).

A comparison of patients with head injuries between the preCOVID-19 and COVID-19 eras is detailed in Table 1. Head injuries were more frequent in pediatric patients $(<3$ years $)$ in the COVID-19 era than in the pre-COVID-19 era (16 of 181 [8.8\%] vs. 6 of 169 [3.6\%], $p=0.048)$. The reasons for 22 pediatric head injuries ( $<3$ year) were as follows : MVC $(n=1)$, dropped by parents $(n=16)$, and slip and fall $(n=5)$. Drop injuries $(<3$ years) occurred more frequently in the COVID-19 era than in the preCOVID-19 era (14 of 16 [87.5\%] vs. 3 of 6 [50.0\%], $p=0.100$ ). MVC injuries occurred in 44 patients $(26.0 \%)$ in the pre-COVID-19 era, and 52 patients (38.7\%) in the COVID-19 era; there was no statistically significant difference between the two injury types $(p=0.632)$.

Table 2 shows a comparison of patients who underwent surgery with those who refused surgery among patients with severe head injuries requiring surgery. Patients who refused surgery were significantly older than those who underwent surgery $(67.7 \pm 14.5$ vs. $52.4 \pm 19.1, p<0.001)$. Married patients refused surgery more frequently than unmarried patients (28 of 33 [84.8\%] vs. 25 of 41 [61.0\%], $p=0.037$ ). Unemployed patients refused surgery more frequently than employed patients (17 of 33 [57.6\%] vs. 13 of 41 [31.7\%], $p=0.034)$. In the pre-COVID-19 era, 25 patients $(69.4 \%)$ underwent surgery, and 11 patients (30.6\%) refused surgery. In the COVID-19 era, 16 patients (42.1\%) underwent surgery, and 22 patients (57.9\%) re-fused surgery. There was a statistically significant difference between the two periods ( $p=0.021)$. Patients who refused surgery had significantly lower GCS scores than those who underwent surgery (6.12 \pm 3.08 vs. $10.6 \pm 3.80, p<0.001)$.

The multivariable logistic regression analysis for factors affecting refusal of surgery among patients with severe head injuries requiring surgery, including old age, marital status, employment status, and COVID-19 era, showed that old age (adjusted odds ratio [OR], 1.076; 95\% confidence interval [CI], 1.033-1.120; $p=0.002$ ), COVID-19 era (adjusted OR, 6.869; 95\% CI, 1.624-29.054; $p=0.009$ ), and lower GCS scores (adjusted OR, 0.694; 95\% CI, 0.568-0.848; $p<0.001)$ were independently associated with refusal of surgery in patients with severe head injuries requiring surgery (Table 3 ).

\section{DISCUSSION}

The COVID-19 pandemic and subsequent social distancing and nation-wide lock-downs to cope with the spread of SARSCoV-2 have impacted almost every area of life ${ }^{1-6,8)}$. Recent studies have reported that the number of trauma cases have decreased during the COVID-19 pandemic ${ }^{3,5,13)}$. Other studies have reported that the spectrum of trauma has shifted from high-energy trauma such as road traffic accidents to low-energy trauma such as house-hold injuries ${ }^{2,5)}$. These results have been reported to be associated with social distancing and nation-wide lockdowns. The purpose of this study was to evaluate the effect of the COVID-19 pandemic on patients with head injuries and their management at a regional emergency medical center in South Korea.

A previous study reported that the prevalence of trauma in children aged $<20$ years was reduced by nearly two-thirds during the COVID-19 pandemic, with a significant reduction 
due to decreased sports injuries ${ }^{1)}$. In our study, head injuries in patients $<20$ years were not significantly different in the preCOVID-19 and COVID-19 eras. However, our results indicated that there was an increase in the number of pediatric patients ( $<3$ years) with head injuries in the COVID-19 era $(8.8 \%$ vs. $3.6 \%, p=0.048)$. Although the statistical significance was not significant $(p=0.100)$, pediatric drop injuries ( $<3$ years) occurred more frequently in the COVID-19 era than in the pre-COVID-19 era (87.5\% vs. 50.0\%). Our findings that pediatric head injuries ( $<3$ years) increased during the COVID-19 pandemic may be partially explained by the increased amount of time spent indoors, resulting in head injuries as the parents held and dropped their children.

Patients with severe head injuries have a high mortality and morbidity ${ }^{7,11,15)}$. Previous studies have proven the relationship between high mortality and lower GCS scores, pupil dilatation, and old age ${ }^{7}$. Long-term outcomes of surgeries for patients with severe head injuries have been reported to be associated with unfavorable outcomes and disabilities; therefore, it is difficult to decide whether to perform surgery in patients with poor prognosis. Patients with severe head injuries are unconscious and lack decision-making capacity; therefore, they depend on their family to decide whether to undergo surgery $^{12,16)}$.

Current trends in decisions making regarding life-sustaining treatments focus on the medical futility and patient's wishes, especially in those with poor prognosis ${ }^{16)}$. Recently, an increasing number of patients and their families want to discontinue life-sustaining treatments when medical futility is expected; however, there are few studies on the topic of discontinuing life-sustaining treatments, such as deciding whether to undergo surgery in patients with severe head injuries.

This study showed that among the 74 patients with severe head injuries requiring surgery, 33 (44.6\%) refused surgery, which was a considerable number. In addition, the rate of refusing surgery increased significantly in the COVID-19 era compared to pre-COVID-19 era (57.9\% vs. $30.6 \%, p=0.021)$. Our study suggests that discontinuation of life-supporting treatment is increasing in patients with severe head injuries and the COVID-19 pandemic is influencing the increase.

In univariate analysis of refusal of surgery, old age and lower GCS scores were associated with refusal of surgery, which are poor prognostic factors of severe head injuries in previous studies. Families of patients with poor prognostic factors, such as old age and lower GCS scores, tended to refuse surgery more often. In our study, married patients refused surgery more frequently than unmarried patients $(84.8 \%$ vs. $61.0 \%$, $p=0.037$ ), which may be partially explained by the rapid decision-making by family. The decision for unmarried patients was generally made by relatives, such as siblings, which delayed the decision on whether to undergo surgery, while the decision for married patients was made faster, because the spouse, son or daughter made the decision. Our results also showed that unemployed patients had a higher rate of refusal of surgery than employed patients ( $68.3 \%$ vs. $42.4 \%, p=0.034$ ). This may be explained by the severity of the economic burden of surgery on unemployed patients with severe head injuries.

In our study, multivariable logistic regression analysis revealed that the COVID-19 pandemic was significantly influencing the treatment planning decisions in patients with severe head injuries (adjusted OR, 6.869). Our results revealed that refusal of surgery in patients with severe head injuries was associated with old age, unemployment, and lower GCS scores. The COVID-19 pandemic and subsequent social distancing and lock-downs may have added an economic burden to the treatment planning decisions in patients with severe head injuries, persuading them to refuse surgery.

Higher rate of refusing surgery among patients with severe head injuries requiring surgery during the COVID-19 pandemic may have been affected by the shift in hospital policy that restricts the number of families allowed in the hospital, including emergency rooms and intensive care units, to prevent the spread of the virus. When more than one family member is involved in decision-making, families tend to opt for surgery in order to avoid the worst case that may result in other family members blaming each other. On the contrary, when the number of family member is restricted to only one, the decision is made faster, thus making it more likely to refuse surgery. However, it is still possible that other causes, such as recent trends over time or regional characteristics, also might affect patient's family to decide not to undergo surgery.

Our study has several limitations. First, this study has a retrospective design with an attendant selection bias. However, the selection bias may be low because all patients with head injuries during the study period in our hospital were included. Second, this study was performed in a single center in Korea, and the generalizability of the results may be limited. The 
types of trauma and the factors that need to be considered during the treatment of head injuries may vary by institution and country. Studies on the recent increase in refusal of surgery in patients with severe head injuries are necessary. This study is meaningful in that it is the first study on this topic. Further studies with larger sample sizes are needed to confirm these preliminary results.

\section{CONCLUSION}

Pediatric head injuries ( $<3$ years) occurred more frequently during the COVID-19 pandemic, possibly due to social distancing and isolation. Patients with severe head injuries are likely to refuse surgery, probably due to the economic burden of the COVID-19 pandemic and subsequent social distancing and lockdowns. Further studies with larger sample sizes are needed to confirm these preliminary results.

\section{CONFLICTS OF INTEREST}

No potential conflict of interest relevant to this article was reported.

\section{INFORMED CONSENT}

This type of study does not require informed consent.

\section{AUTHOR CONTRIBUTIONS}

\author{
Conceptualization : $\mathrm{SHK}$ \\ Data curation : TMN, JHJ \\ Formal analysis : DHK \\ Funding acquisition : KHK \\ Methodology : YZK \\ Project administration : KHK \\ Visualization : TMN \\ Writing - original draft : TMN \\ Writing - review \& editing : SHK
}

\section{ORCID}

$\begin{array}{ll}\text { Taek Min Nam } & \text { https://orcid.org/0000-0002-9596-4659 } \\ \text { Do-Hyung Kim } & \text { https://orcid.org/0000-0002-4900-7049 } \\ \text { Ji Hwan Jang } & \text { https://orcid.org/0000-0001-6364-3992 } \\ \text { Young Zoon Kim } & \text { https://orcid.org/0000-0003-1171-0780 } \\ \text { Kyu Hong Kim } & \text { https://orcid.org/0000-0002-5027-4881 } \\ \text { Seung Hwan Kim } & \text { https://orcid.org/0000-0002-5898-6517 }\end{array}$

\section{References}

1. Baxter I, Hancock G, Clark M, Hampton M, Fishlock A, Widnall J, et al. : Paediatric orthopaedics in lockdown: a study on the effect of the SARSCov-2 pandemic on acute paediatric orthopaedics and trauma. Bone Jt Open $1: 424-430,2020$

2. Devarakonda AK, Wehrle CJ, Chibane FL, Drevets PD, Fox ED, Lawson $A G$ : The effects of the COVID-19 pandemic on trauma presentations in a level one trauma center. Am Surg 87 : 686-689, 2021

3. Goyal N, Swain SK, Gupta K, Chaturvedi J, Arora RK, Sharma SK : "Locked up inside home" - Head injury patterns during coronavirus disease of 2019 pandemic. Surg Neurol Int 11 : 395, 2020

4. Hampton M, Clark M, Baxter I, Stevens R, Flatt E, Murray J, et al. : The effects of a UK lockdown on orthopaedic trauma admissions and surgical cases: a multicentre comparative study. Bone Jt Open 1 : 137-143, 2020

5. Hashmi PM, Zahid M, Ali A, Naqi H, Pidani AS, Hashmi AP, et al. : Change in the spectrum of orthopedic trauma: effects of COVID-19 pandemic in a developing nation during the upsurge; a cross-sectional study. Ann Med Surg (Lond) 60 : 504-508, 2020

6. Kuitunen I, Ponkilainen VT, Launonen AP, Reito A, Hevonkorpi TP, Paloneva J, et al. : The effect of national lockdown due to COVID-19 on emergency department visits. Scand J Trauma Resusc Emerg Med $28: 114,2020$

7. Lan Z, Richard SA, Li Q, Wu C, Zhang Q, Chen R, et al. : Outcomes of patients undergoing craniotomy and decompressive craniectomy for severe traumatic brain injury with brain herniation: a retrospective study. Medicine (Baltimore) 99 : e22742, 2020

8. Mason TB, Barrington-Trimis J, Leventhal AM : Eating to cope with the COVID-19 pandemic and body weight change in young adults. J Adolesc Health 68 : 277-283, 2021

9. Min J, Kim HW, Koo HK, Ko Y, Oh JY, Kim J, et al. : Impact of COVID-19 pandemic on the national PPM tuberculosis control project in Korea: the Korean PPM monitoring database between July 2019 and June 2020. J Korean Med Sci 35 : e388, 2020

10. Na KR, Kim HR, Ham Y, Choi DE, Lee KW, Moon JY, et al. : Acute kidney injury and kidney damage in COVID-19 patients. J Korean Med Sci 35 : e257, 2020

11. Quintard H, Lebourdon $X$, Staccini $P$, Ichai C : Decompression surgery 
for severe traumatic brain injury (TBI): a long-term, single-centre experience. Anaesth Crit Care Pain Med 34 : 79-82, 2015

12. Smirdec M, Jourdain M, Guastella V, Lambert C, Richard JC, Argaud L, et al. : Impact of advance directives on the variability between intensivists in the decisions to forgo life-sustaining treatment. Crit Care $24: 672$, 2020

13. Sugand K, Park C, Morgan C, Dyke R, Aframian A, Hulme A, et al. : Impact of the COVID-19 pandemic on paediatric orthopaedic trauma workload in central London: a multi-centre longitudinal observational study over the "golden weeks". Acta Orthop 91 : 633-638, 2020
14. Suh HJ, Kim DH, Heo EY, Lee HW, Lee JK, Lee CS, et al. : Clinical characteristics of COVID-19: clinical dynamics of mild severe acute respiratory syndrome coronavirus 2 infection detected by early active surveillance. J Korean Med Sci 35 : e297, 2020

15. Tian R, Dong J, Liu W, Zhang J, Han F, Zhang B, et al. : Prognostic analysis of emergency decompressive craniectomy for patients with severe traumatic brain injury with bilateral fixed dilated pupils. World Neurosurg 146 : e1307-e1317, 2021

16. Van Norman GA : Decisions regarding forgoing life-sustaining treatments. Curr Opin Anaesthesiol 30 : 211-216, 2017 